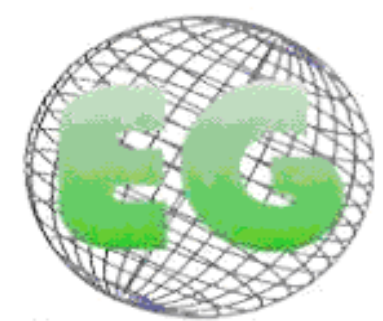

ISSN 1695-6141

www.um.es/egloball

\title{
CLÍNICA
}

\section{Plantas medicinales utilizadas en la salud infantil}

Plantas medicinais utilizadas na saúde da criança

\section{*Souza, ADZ., " ${ }^{* *}$ Ceolin, T., ${ }^{* * *}$ Vargas, NRC., ${ }^{* * * * H e c k, ~ R M ., ~}{ }^{* * * *}$ Vasconcellos, CL., ${ }^{* * * * * B}$ Borges, AM., ${ }^{* * * * * *}$ Mendieta, MC.}

*Estudiante del $9^{\circ}$ semestre de la Facultad de Enfermería (FEn) /Universidad Federal de Pelotas (UFPel). Bolsista de iniciación científica por el CNPq. ${ }^{* *}$ Mestre em Enfermagem. Profa Asistente de la FEn/UFPel. ***Estudiante del $8^{\circ}$ semestre de la FEn/UFPel. Bolsista de iniciación científica FAPERGS. ${ }^{* * * * D o c t o r a ~ e n ~}$ Enfermería. Profesora Adjunta de la FEn/UFPel. ${ }^{* * * * *}$ Mestre en Enfermería por el Programa de PosGraduación en Enfermería de la Fen/UFPel. ${ }^{* * * * * *}$ Estudiante del $7^{\circ}$ semestre de la Facultad de Enfermería (FEn)/Universidade Federal de Pelotas (UFPel). Bolsista de iniciación científica por el CNPq.

(Investigación financiada por el Consejo Nacional de Desarrollo Científico y Tecnológico/Brasil)

Palabras clave: Plantas medicinales; Enfermería en salud comunitaria; Salud del niño; Terapias complementarias

Palavras-chave: Plantas medicinais; Enfermagem em saúde comunitária; Saúde da criança; Terapias complementares

Keywords: Medicinal plants; Community health nursing; Child health; Complementary therapies

\section{RESUMEN}

Identificar las plantas medicinales utilizadas en la salud infantil por familias de agricultores de base ecológica de la región Sur de Río Grande do Sul, Brasil. El enfoque de investigación es cualitativo, exploratorio y descriptivo, con datos recogidos de enero a mayo de 2009. Los sujetos fueron ocho familias de agricultores, residentes en los municipios de Pelotas, Morro Redondo, Canguçu y Arroio do Padre. Seis plantas fueron citadas (Chrysanthemun cinerariifolium, Foeniculum vulgare, Acca sellowiana, Citrus sp. Origanun sp. Sambucus spp.), y sólo de una (Foeniculum vulgare) encontramos estudios farmacológicos comprobando lo que los sujetos de la investigación referían. Es muy importante que el profesional enfermero tenga conocimientos sobre plantas medicinales para transmitir la información pertinente sobre la forma de preparación, dosis e indicaciones relativas a las plantas utilizadas en la salud infantil. 


\section{RESUMO}

Identificar as plantas medicinais utilizadas na saúde da criança, por famílias de agricultores de base ecológica da Região Sul do Rio Grande do Sul, Brasil. A pesquisa é de abordagem qualitativa, exploratória e descritiva, sendo os dados coletados de janeiro a maio de 2009. Os sujeitos foram de oito famílias de agricultores, residentes nos municípios de Pelotas, Morro Redondo, Canguçu e Arroio do Padre. Foram citadas seis plantas (Chrysanthemun cinerariifolium, Foeniculum vulgare, Acca sellowiana, Citrus sp., Origanun sp., Sambucus spp.), sendo que apenas para uma (Foeniculum vulgare) encontramos estudos farmacológicos comprovando o que os sujeitos da pesquisa referiram. É de extrema importância que o profissional enfermeiro tenha conhecimento a respeito das plantas medicinais para transmitir informações pertinentes sobre a forma de preparo, dosagens e indicações relacionados às plantas utilizadas na saúde da criança.

\section{ABSTRACT}

The aim was to identify medicinal plants used in child health, for family farmers and the ecological base of the southern Rio Grande do Sul, Brazil. The research approach is qualitative, exploratory and descriptive, with data collected from January to May 2009. The subjects were eight families of farmers, residents in the cities of Pelotas, Morro Redondo, Canguçu and Arroyo do Padre. Six plants were cited (Chrysanthemun cinerariifolium, Foeniculum vulgare, Acca sellowiana, Citrus sp. Origanun sp. Sambucus spp.), and only for one (Foeniculum vulgare) were pharmacological studies found that corroborated what the subjects mentioned. It is extremely important that the nurse has knowledge of medicinal plants to transmit relevant information about the preparation, dosages and indications related to plants used in child health.

\section{INTRODUCCIÓN}

Desde tlempos remotos, el ser humano ha buscado conocer las plantas medicinales, pues además de ser utilizadas en la prevención de patologías, son de fácil acceso a la población, menos agresivas para la salud, causando menores efectos secundarios ${ }^{1}$, convirtiéndose en una importante herramienta para la realización del cuidado integral de la salud ${ }^{2}$.

Las plantas medicinales son muy utilizadas por la población y transmitidas de generación a generación, incluso en el cuidado de la salud del niño, donde las costumbres, como los tés caseros, aún están muy presentes. En el proceso del cuidado es esencial que se conozca cómo se procesa el crecimiento, el desarrollo, las necesidades básicas del niño, los riesgos a los que está sometido y algunos cuidados para la preservación de la salud, contribuyendo a la disminución de gastos y tecnologías en el tratamiento de enfermedades capaces de ser prevenidas $^{3}$.

En ese contexto, se evidencia la importancia del profesional enfermero, pues es él el que acompaña el crecimiento y el desarrollo del niño a través de la puericultura; apoya y orienta a la familia, comprendiendo los efectos de determinantes culturales, sociales y ambientales, interviniendo de forma apropiada para mantener la salud del niño ${ }^{4}$, incluso a través de las orientaciones sobre el uso adecuado de plantas medicinales, como dosificación y contraindicaciones.

El Consejo Federal de Enfermería (COFEn)/Brasil, a través del Parecer Normativo n.004/95, reconoció que las terapias alternativas/complementarias (acupuntura, iridología, fitoterapia, reflexología, quiropraxia y masoterapia, entre tantas otras) son prácticas originadas, en su mayoría, de culturas orientales, no estando vinculadas a ninguna categoría profesional. La Resolución 197/97 del COFEn establece y reconoce las terapias alternativas/complementarias como especialidad y/o calificación del enfermero, desde que haya concluido y haya sido aprobado en el curso o entidad similar, con una carga horaria mínima de 360 horas $^{5}$. 
Ante esa perspectiva y ante las críticas en relación al modelo biomédico basado, exclusivamente, en el uso de medicamentos industrializados, surge la necesidad de acciones dirigidas a la mejora de las condiciones de salud de la población y de la calidad de vida, por lo que el gobierno federal viene incentivando el uso de terapias complementarias en el Sistema Único de Salud (SUS). En 2006, el Ministerio de la Salud implantó la Política Nacional de Prácticas Integradas y Complementarias (PNPIC) en el SUS, incentivando el uso de las plantas medicinales, a través de la fitoterapia, homeopatía, acupuntura, entre otras prácticas ${ }^{6}$. Esta política, no atribuyó a ninguna categoría profesional específica la indicación para el uso terapéutico de las plantas medicinales, volviéndose así, una amplia área de actuación a ser explorada por los enfermeros.

Este trabajo tiene el objetivo de identificar las plantas medicinales utilizadas en la salud del niño por los agricultores de base ecológica en la región Sur de Rio Grande do Sul.

\section{METODOLOGÍA}

Se trató de un estudio cualitativo ${ }^{7}$. La investigación está vinculada al proyecto Plantas Bioactivas de uso humano por familias de agricultores de base ecológica en la región Sur del RS, desarrollado por la Facultad de Enfermería de la Universidad Federal de Pelotas y por la Embrapa Clima Temperado. Los sujetos se constituyeron por agricultores de base ecológica, que fueron seleccionados a través de la indicación del coordinador de la asociación de los feriantes $^{8}$, como conocedores de plantas medicinales. Además de los 8 agricultores indicados, fueron abordadas sus generaciones familiares, correspondiendo a un total de 19 sujetos, siendo por lo menos dos generaciones en cada familia. Los encuestados comercializan sus productos en la feria ecológica de Pelotas, que ocurre cuatro veces por semana, en locales distintos de la ciudad.

El lugar de estudio fue el domicilio de los agricultores, situado en el área rural de los municipios de Pelotas, Morro Redondo, Canguçu y Arroio do Padre, en la región Sur de Rio Grande do Sul/Brasil. La recolección de datos sobre las plantas medicinales utilizadas, destacando las plantas utilizadas en la salud del niño, ocurrió entre enero y mayo de 2009.

Fueron utilizados los siguientes instrumentos: encuesta semiestructurada ${ }^{9}$, observación sistemática ${ }^{10}$ de las plantas con registro fotográfico y el georreferenciamiento, realizado a través del Sistema de Posicionamiento Global (GPS). El georreferenciamiento fue utilizado con el objetivo de identificar la localización de las plantas investigadas, lo que permite que otro investigador localice geográficamente, con exactitud, determinada planta.

Se realizó la recolección de exsicatas para algunas plantas medicinales. Las plantas recolectadas y/o fotografiadas fueron identificadas por un botánico, vinculado a la Embrapa Clima Temperado. Fueron respetados los principios éticos de investigaciones con seres humanos. El proyecto recibió aprobación del Comité de Ética e Investigación de la Facultad de Medicina de la Universidad Federal de Pelotas (072/2007).

En relación al análisis de los datos, las informaciones correspondientes al conocimiento de los agricultores sobre las plantas medicinales utilizadas en la salud del niño fueron expuestas en una tabla y posteriormente comparadas con estudios farmacológicos y fitoquímicos. 


\section{RESULTADOS}

Fueron abordados 19 encuestados, entre ellos dieciséis (84\%) eran del sexo femenino. En relación a la distribución etaria de los encuestados, ocho $(42,7 \%)$ se encontraban entre los 20 y los 39 años. La forma predominante de preparación de las plantas fue la infusión, que consiste en derramar agua hirviendo sobre la planta medicinal y, en seguida, tapar el recipiente por un período de tiempo determinado ${ }^{11}$.

Tabla 1 - Plantas medicinales utilizadas por los encuestados para la salud del niño. Pelotas, Rio Grande do Sul, Brasil, 2011.

\begin{tabular}{llll}
\hline $\begin{array}{l}\text { Nombre } \\
\text { científico }\end{array}$ & Família & $\begin{array}{l}\text { Nombre } \\
\text { popular }\end{array}$ & Uso mencionado por los entrevistados \\
\hline $\begin{array}{l}\text { Chrysanthemun } \\
\text { cinerariifolium }\end{array}$ & Asteraceae & manzanilla & $\begin{array}{l}\text { Para aliviar el dolor de estómago y cólico } \\
\text { del bebé, disminuir problemas en el }\end{array}$ \\
& & $\begin{array}{l}\text { hígado y para lavar los ojos. Utilizada } \\
\text { como calmante. Muchacha no puede }\end{array}$ \\
& & $\begin{array}{l}\text { tomar, pues perjudica el útero. Tiene } \\
\text { efecto abortivo. }\end{array}$
\end{tabular}

$\begin{array}{lll}\begin{array}{l}\text { Foeniculum Apiaceae } \\ \text { vulgare }\end{array} & \text { Usado para disminuir gases, disminuir } \\ & \text { cólicos en bebés, disminuir dolor de } \\ & \text { estómago y aliviar las molestias de la } \\ \text { barriga hinchada. }\end{array}$

Acca Myrtaceae guayabo Para tratar los síntomas del rotavirus, sellowiana disminuir los cólicos y diarrea.

Citrus sp. Rutaceae lima Para tratar hipertermia de los bebés.

Origanun sp. Lamiaceae mejorana Disminuir cólicos intestinales en los mayores y en los bebés, y disminuir cólico menstrual. En grandes cantidades es abortiva.

Sambucus spp. Caprifoliaceae saúco

En tratamiento de sarampión y para bajar la fiebre. Tratar "sarpullidos" de los bebés. La fruta puede ser utilizada como laxante.

En cuanto a la localización, las plantas se encontraban en el huerto, en la quinta, en el jardín y en el campo, siendo que la mayor parte de ellas estaba próxima a las viviendas de los agricultores. La mayoría de los encuestados, en relación a la dosificación, refirió que siempre que preparaban los tés para los niños, era en dosis menor y con mayor dilución, comparados con la preparación de tés para los mayores.

\section{DISCUSIÓN}

Al analizar el uso de las plantas medicinales en el cuidado de la salud del niño, destacamos un área donde el enfermero se puede calificar, siendo que cada vez más el Ministerio de la Salud viene incentivando el uso de las terapias complementarias en el SUS, a través de proyectos de investigación, capacitación y cursos ${ }^{12}$. Para obtener subsidios de trabajo con la educación en salud sobre plantas medicinales, ese profesional debe instrumentalizarse a 
través de la revisión de estudios científicos que puedan dar base a las informaciones y a las necesidades de cuidado del usuario en relación al uso de las plantas medicinales de manera que complementen el tratamiento alopático.

La promoción de la salud tiene estrecha relación con la educación en salud, estableciendo cambios de saberes entre profesional y usuario, a partir de la participación de la población, conociendo sus necesidades, estilo de vida, creencias, valores y el contexto cultural y sociopolítico en que viven ${ }^{13}$. Con eso percibimos la importancia de que el enfermero mantenga una buena relación con la población, conociendo el territorio donde actúa, para que consiga orientar y dialogar de acuerdo a la realidad local.

En esa perspectiva, buscamos estudios farmacológicos que comprobaran los efectos de las plantas medicinales citados por los encuestados y utilizados en el cuidado con la salud del niño. Encontramos para la Chrysanthemun cinerariifolium, un estudio mostrando una planta del mismo género, la Chrysanthemun coranarium, en ella fueron aisladas proteínas antifúngicas de semillas, que inhibieron la actividad del virus de la inmunodeficiencia humana -1 transcriptase reversa ${ }^{14}$.

Se sabe que en Brasil, debido a las distintas características climáticas y a la diversidad de la flora, muchas plantas reciben el mismo nombre popular en diferentes localidades, aunque siendo especies diferentes y con principios activos distintos ${ }^{12}$.

Observamos durante la recolección de datos, que muchas veces ocurría una confusión con los encuestados en la identificación de la manzanilla, siendo utilizada la Chrysanthemun cinerariifolium cuando buscaban el efecto de la Chamomilla recutita, la que poseé propiedades inmunoestimulantes, espasmolíticas, bacterisotáticas, ansiolíticas y sedativas ${ }^{15}$. Para la Chrysanthemun parthenium, del mismo género de la Chrysanthemun cinerariifolium, fueron encontrados efectos estomacales, calmantes y carminativos ${ }^{16}$.

En relación al Foeniculum vulgare, ensayos realizados en laboratorios mostraron actividades insecticidas, antifúngicas, digestivas, carminativas y espasmolíticas. Un estudio aleatorizado, doble ciego mostró que cólicos en lactantes mejoran en una semana de tratamiento con un extracto con base Chamomilla recutita, Foeniculum vulgare y Melissa officinalis ${ }^{18}$, mostrando que los estudios farmacológicos van al encuentro de la utilización referida por los agricultores. Sobre la Acca sellowiana, no fue encontrado ningún estudio farmacológico comprobando el efecto referido.

Estudios etnofarmacológicos evidenciaron que las hojas del Origanun sp. son utilizadas en el tratamiento de la gripe, del resfriado, de la indigestión, de la flatulencia, de los problemas estomacales y de los cólicos menstruales ${ }^{15}$. No encontramos estudios farmacológicos; sin embargo, en otras investigaciones basadas en el conocimiento popular fueron encontradas, los mismos efectos que los encuestados han referido.

El Citrus sp., posee acción antiinflamatoria y protectora de los capilares sanguíneos ${ }^{15}$. Estudios con varias plantas, entre ellas el Sambucus nigra, mostraron una reducción significativa en el peso corporal en humanos y animales, mostrando eficacia en la lucha contra la obesidad $^{19}$. En relación al Citrus sp. y al Sambucus spp. no fueron encontrados estudios farmacológicos que comprobaran la eficacia citada por los encuestados.

Entre las seis plantas medicinales referidas por los agricultores, solo en una (Foeniculum vulgare) se encontraron estudios farmacológicos que comprobaran los efectos citados por los encuestados de la investigación, resaltando la necesidad de ampliación de las 
investigaciones farmacológicas sobre las plantas medicinales que investigan la amplia diversidad de nuestra flora brasileña.

Entre las plantas referidas por los agricultores ecológicos, dos (Foeniculum vulgare/ Chamomilla recutita) forman parte de la Relación Nacional de Plantas Medicinales de Interés al SUS (RENISUS) ${ }^{20}$, donde están presentes 71 plantas, con el objetivo de orientar estudios que puedan subsidiar la elaboración de la relación de fitoterápicos disponibilizados por el Ministerio de la Salud para el uso de la población.

Resaltamos que existen pocos estudios relacionados con esta temática, por eso son importantes investigaciones sobre las plantas medicinales, ya que el niño necesita estar acompañado a través de un cuidado integral que considere el saber y el contexto donde él y su familia están insertados.

\section{CONSIDERACIONES FINALES}

Existen pocos estudios relacionados con las plantas referidas por los encuestados, información sobre la dosificación, la forma de preparación y la cantidad utilizada en los niños. Por tanto, existe la necesidad de la realización de más investigaciones en esa área, así como una mayor atención en la administración de las plantas en los niños, ya que una planta medicinal usada incorrectamente puede ser perjudicial para la salud.

El enfermero tiene un papel fundamental en la gestión del cuidado, pues está constantemente acompañando a la población en las instituciones de salud; por eso es importante que se capacite sobre las plantas medicinales, pudiendo informar a la comunidad sobre los beneficios y perjuicios a través de la educación en salud, objetivando la promoción de la salud y la prevención de enfermedades.

\section{REFERENCIAS}

1. Di Stasi LC. Plantas medicinais verdades e mentiras - O que os usuários e os profissionais de saúde precisam saber. São Paulo: UNESP; 2007.

2. Ceolin $\mathrm{T}$, et al. Inserción de terapias complementarias en el sistema único de salud atendiendo al cuidado integral en la asistencia. Revista Enfermería Global. 2009 [acesso em 2010 out. 11]; 16: 1-10. Disponível em: http://revistas.um.es/eglobal/article/view/66311/63931 3. Carvalho A, Salles F, Guimarães M, Armond L, organizadores. Saúde da criança. Belo Horizonte: UFMG; 2002.

4. Brasil. Ministério da Saúde. Saúde da criança: acompanhamento do crescimento e desenvolvimento infantil. Brasília: Ministério da Saúde; 2002.

5. Conselho Federal de Enfermagem (COFEn). Resolução 197/1997. Dispõe sobre as terapias alternativas. [Acesso em: 2009 jun 5] Disponível em: http://www.portalcofen.gov.br/2007/materias.asp?ArticleID=7041\&sectionID=34

6. Brasil. Ministério da Saúde. Secretaria de Atenção à Saúde. Departamento de Atenção Básica. Política Nacional de Práticas Integrativas e Complementares no SUS - PNPIC-SUS. Brasília: Ministério da Saúde; 2006.

7. Minayo MCS. O desafio do conhecimento: pesquisa qualitativa em saúde. 11ª ed. São Paulo - Rio de Janeiro: HICITEC-ABRASCO; 2008.

8. Goodman LA. Snowball Sampling. Annals of Mathematical Statistics. 1961; (32):148-70.

9. Triviños ANS. Introdução à pesquisa em ciências sociais - A pesquisa qualitativa em educação. São Paulo: Atlas; 2008.

10. Gil AC. Métodos e técnicas de pesquisa social. 5aㅡ ed. São Paulo: Atlas; 2007. 
11. Brasil. Agência Nacional de Vigilância Sanitária (ANVISA). Resolução - RDC no 10, de 9 de março de 2010 [acesso em 2010 Jun. 12]. Disponível em: http://www.brasilsus.com.br/legislacoes/rdc/103202-10

12. Ceolin T, Heck RM, Barbieri RL, Souza ADZ, Rodrigues WF, Vanini M. Plantas medicinais utilizadas como calmantes por agricultores ecológicos da região Sul do Rio Grande do Sul, Brasil. Rev. enferm. UFPE on line. 2009 [acesso em 2010 out. 11]; 3(4):25360.

Disponível

http://www.ufpe.br/revistaenfermagem/index.php/revista/article/view/116/116

13. Santos RV, Penna CMM. A educação em saúde como estratégia para o cuidado à gestante, puérpera e ao recém-nascido. Texto \& contexto enferm. 2009; 18(4): 652-660.

14. Wang $\mathrm{H}, \mathrm{Ye} X Y, \underline{\mathrm{Ng} T B}$. Purification of chrysancorin, a novel antifungal protein with mitogenic activity from garland chrysanthemum seeds. Biol. chem. 2001; 6(382): 947-51.

15. Lorenzi H, Matos FJA. Plantas Medicinais no Brasil - Nativas e Exóticas. Nova Odessa (SP): Instituto Plantarum; 2008.

16. Matos FJA. Farmácias vivas: sistema de utilização de plantas medicinais - projeto para pequenas comunidades. 4 $4^{\mathrm{a}}$ ed. Fortaleza: Editora UFC; 2002.

17. Simões CMO, et al. Plantas da Medicina Popular no Rio Grande do Sul. Porto Alegre (RS): UFRGS; 1998.

18. Savino F, Cresi F, Castagno E, Silvestro L, Oggero R. A randomized double-blind placebo-controlled trial of a standardized extract of Matricariae recutita, Foeniculum vulgare and Melissa officinalis (ColiMil) in the treatment of breastfed colicky infants. Phytother. res. 2005; 4(19): 335-40.

19. Hasani RS, Nayebi $\mathrm{N}$, Larijani $\mathrm{B}$, Abdollahi M. A systematic review of the efficacy and safety of herbal medicines used in the treatment of obesity. World j. gastroenterol. 2009; 25 (15): 3073-85. 20.

20. Brasil. Ministério da Saúde. RENISUS - Relação Nacional de Plantas Medicinais de Interesse ao SUS [homepage na internet]. Agencia Brasil. [atualizada em 2009 Mar 8; acesso em: 2009 Maio 29]. Disponível em: http://portal.saude.gov.br/portal/arquivos/pdf/RENISUS.pdf

ISSN 1695-6141

๑) COPYRIGHT Servicio de Publicaciones - Universidad de Murcia 